Proyecciones

Vol. 20, No 1, pp. 83-91, May 2001.

Universidad Católica del Norte

Antofagasta - Chile

\title{
ON THE LEVI PROBLEM WITH SINGULARITIES
}

\author{
ALAOUI YOUSSEF \\ Institute Agronomique et Vétérinaire, Maroc
}

\begin{abstract}
In section 1, we show that if $X$ is a Stein normal complex space of dimension $n$ and $D \subset \subset X$ an open subset which is the union of an increasing sequence $D_{1} \subset D_{2} \subset \ldots \subset D_{n} \subset \subset \ldots$ of domains of holomorphy in $X$, then $D$ is a domain of holomorphy.

In section 2, we prove that a domain of holomorphy $D$ which is relatively compact in a 2-dimensional normal Stein space $X$ itself is Stein.

In section 3, we show that if $X$ is a Stein space of dimension $n$ and $D \subset X$ an open subspace which is the union of an increasing sequence $D_{1} \subset D_{2} \subset \ldots \subset D_{n} \subset \ldots$ of open Stein subsets of $X$, then $D$ itself is Stein, if $X$ has isolated singularities.
\end{abstract}




\section{Introduction}

Is a complex space $\mathrm{X}$ which is the union of an increasing sequence $X_{1} \subset X_{2} \subset X_{3} \subset \cdots$ of open Stein subspaces itself a Stein space?

From the begining this question has held great interest in Stein theory.

The special case when $\left\{X_{j}\right\}_{j \geq 1}$ is a sequence of Stein domains in $\mathbb{C}^{n}$ had been proved long time ago by Behnke and Stein [2].

In 1956, Stein [13] answered positively the question under the additional hypothesis that $\mathrm{X}$ is reduced and every pair $\left(X_{\nu+1}, X_{\nu}\right)$ is Runge.

In the general case $\mathrm{X}$ is not necessarily holomorphically-convex.

Fornaess [7], gave a 3-dimensional example of such situation.

In 1977, Markoe [10] proved the following:

Let $\mathrm{X}$ be a reduced complex space which the union of an increasing sequence $X_{1} \subset X_{2} \subset \cdots \subset X_{n} \subset \cdots$ of Stein domains.

Then $\mathrm{X}$ is Stein if and only if $H^{1}\left(X, O_{X}\right)=0$.

M. Coltoiu has shown in [3] that if $D_{1} \subset D_{2} \subset \cdots \subset D_{n} \subset \cdots$

is an increasing sequence of Stein domains in a normal Stein space X, then $D=\bigcup_{j \geq 1} D_{j}$ is a domain of holomorphy. (i.e. for each $x \in \partial D$ there is $f \in O(D)$ which is not holomorphically extendable through $\mathrm{x})$.

The aim of this paper is to prove the following theorems:

Theorem 1. -Let $X$ be a Stein normal space of dimension $n$ and $D \subset \subset X$ an open subset which the union of an increasing sequence $D_{1} \subset D_{2} \subset \cdots \subset D_{n} \cdots$ of domains of holomorphy in $\mathrm{X}$. Then $\mathrm{D}$ is a domain of holomorphy.

Theorem 2. -A domain of holomorphy D which is relatively compact in a 2-dimensional normal Stein space X itself is Stein

Theorem 3. -Let $\mathrm{X}$ be a Stein space of dimensinon $\mathrm{n}$ and $D \subset X$ an open subspace which is the union of an increasing sequence $D_{1} \subset D_{2} \subset \cdots \subset D_{n} \subset \cdots$ of open Stein subsets of X. Then D itself is Stein, if X has isolated singularities. 


\section{Preliminaries}

It should be remarked that the statement of theorem 2 is in general false if $\operatorname{dim}(X) \geq 3$ :

Let $X=\left\{z \in \mathbb{C}^{4}: z_{1}^{2}+z_{2}^{2}+z_{3}^{2}+z_{4}^{2}=0\right\}, H=\left\{z \in \mathbb{C}^{4} ; z_{1}=i z_{2}, z_{3}=\right.$ $\left.i z_{4}\right\}$,

$U=\{z \in X:|z|<1\}$, and $D=U-U \cap H$.

$\mathrm{X}$ is a Stein normal space of dimension 3 with the singularity only at the origine. Since D is the complement of a hypersurface on the Stein space $\mathrm{U}$, then D is a domain of holomorphy. But D is not Stein. (See $[9])$.

Let $\mathrm{X}$ be a connected $\mathrm{n}$-dimensional, Stein normal space and $\mathrm{Y}$ be the singular locus of $\mathrm{X}$.

There exist finitely many holomorphic maps

$$
\phi_{j}: X \longrightarrow \mathbb{C}^{n}, j=1, \cdots, l
$$

with discrete fibers, and holomorphic functions $f_{1}, \cdots, f_{l}$ on $\mathrm{X}$ such that the branch locus of $\phi_{j}$ is contained in $Z_{j}=\left\{f_{j}=0\right\}$ and $Y=\bigcap_{j=1}^{l} Z_{j}$.

\section{Proofs of theorems}

We prove theorem 1 using the method of Fornaess and Narasimhan [8] (See also lemma 7, [1]).

For every irreducible component $X_{i}$ of $\mathrm{X}, X_{i} \cap D \subset \subset X_{i}$ is an irreducible component of $\mathrm{D}$ and a union of an increasing sequence of domains of holomorphy in the Stein space $X_{i}$. (See [11]).

Since each $X_{i}$ is normal and $\left(X_{i} \cap D\right)_{i}$ are pairwise disjoint domains, then we may assume that $\mathrm{X}$ is connected.

Let $q \in \partial D-Y$ and choose holomorphic functions $h_{1}, \cdots, h_{m}$ on $\mathrm{X}$ such that: $\left\{x \in X / h_{i}(x)=0, i=1, \cdots, m\right\}=\{q\}$, and $\mathrm{j}$ such that $q \notin Z_{j}$.

Since $D-Z_{j}$ is the union of the increasing sequence $\left(D_{k}-Z_{j}\right)_{k \geq 1}$ of the Stein sets $D_{k}-Z_{j}$ in the Stein manifold $X-Z_{j}$, then $D-Z_{j}$ is Stein. Let $d_{j}$ be the boundary distance of the unramified domain 
$\phi_{j}: D-Z_{j} \longrightarrow \mathbb{C}^{n}$. Then $-\log d_{j}$ is plurisubharmonic on $D-Z_{j}$. Therefore the function

$$
\psi_{j}(z)=\left\{\begin{array}{l}
\operatorname{Max}\left(0,-\log d_{j}+k_{j} \log \left|f_{j}\right|\right) \text { on } D-Z_{j} \\
0 \text { on } Z_{j}
\end{array}\right.
$$

is plurisubharmonic on $\mathrm{D}$, if $k_{j}$ is a large constant. This follows from a result due to Oka. (See also [1], lemma 7).

By the Nullstellensatz, There exist a neighborhood V of q in X and constants $c>0, N>0$ such that

$$
\sum_{i=1}^{m}\left|h_{i}(x)\right|^{2} \geq c\left|\phi_{j}(x)-\phi_{j}(q)\right|^{N}, x \in V
$$

Since $\phi_{j}$ is an analytic isomorphism at $\mathrm{q}$ and $f_{j}(q) \mp 0$, it follows that, if $\mathrm{V}$ is sufficiently small, there is a constant $c_{0}>0$ such that

$$
\sum_{i=1}^{m}\left|h_{i}(x)\right|^{2} \geq c_{0} \exp \left(-N \psi_{j}(x)\right), x \in V \cap D .
$$

Now, since $\psi_{j} \geq 0$, there exist constants $c_{1}, c_{2}>0$ such that

$$
c_{2} \exp \left(-N \psi_{j}(x)\right) \leq \sum_{i=1}^{m}\left|h_{i}(x)\right|^{2} \leq c_{1} \exp \left(\psi_{j}(x)\right), x \in D
$$

And applying the theorem of Skoda [14], we deduce that there is a constant $k>0$ and holomorphic functions $g_{1}, \cdots, g_{m}$ on $D-Z_{j}$ such that

$$
\sum_{i=1}^{m} g_{i} h_{i}=1 \text { on } D-Z_{j}
$$

and

$$
\sum_{i=1}^{m} \int_{D-Z_{j}}\left|g_{i}(x)\right|^{2} \exp \left(-k \psi_{j}(x)\right) d v<\infty
$$

Where $\mathrm{dv}$ is Lebesgue measure pulled back to $\mathrm{D}$ and $\mathrm{k}$ depending only on $\mathrm{N}$ and $\mathrm{m}$. The existence of a holomorphic function $\mathrm{f}$ on $\mathrm{D}$ which is unbounded on any sequence $\left\{q_{\mu}\right\}$ of points approaching $\mathrm{q}$ follows from lemma 3-1-2 of Fornaess-Narasimhan [8]. Since $\partial D-Y$ 
is dense in $\partial D$, it follows that $\mathrm{D}$ is a domain of holomorphy.

We shall prove theorem 2 using the following result of R.Simha [15].

theorem 4. -Let X be a normal Stein complex space of dimension 2 , and

$\mathrm{H}$ a hypersurface in $\mathrm{X}$. Then $X-H$ is Stein.

\section{Proof of theorem 2}

By the theorem of Andreotti-Narasimhan [1], it is suffficient to prove that D is locally Stein, and we may of course assume that $\mathrm{X}$ is connected.

Let $p \in \partial D \cap Y$, and choose a connected Stein open neighborhood U of p with $U \cap Y=\{p\}$ and such that $\mathrm{U}$ is biholomorphic to a closed analytic set of a domain $\mathrm{M}$ in some $\mathbb{C}^{N}$. Let $\mathrm{E}$ be a complex affine subspace of $\mathbb{C}^{N}$ of maximal dimension such that $\mathrm{p}$ is an isolated point of $E \cap U$.

By a coordinate transformation one can obtain that $z_{i}(p)=0$ for all $i \in\{1, \cdots, N\}$ and we may assume that there is a connected Stein neighborhood $\mathrm{V}$ of $\mathrm{p}$ in $\mathrm{M}$ such that $U \cap V \cap\left\{z_{1}(x)=z_{2}(x)=0\right\}=\{p\}$. We may, of course, suppose that $N \geq 4$, and let $E_{1}=V \cap\left\{z_{1}(x)=\cdots=z_{N-2}(x)=0\right\}, E_{2}=\left\{x \in E_{1}: z_{N-1}(x)=0\right\}$. Then $A=(U \cap V) \cup E_{1}$ is a Stein closed analytic set in $\mathrm{V}$ as the union of two Stein global branches of A.

Let $\zeta: \hat{A} \rightarrow A$ be a normalization of A. Then $\zeta: \hat{A}-\zeta^{-1}(p) \rightarrow A-\{p\}$ is biholomorphic. Since $\zeta^{-1}\left(E_{1}\right)=\left\{x \in \hat{A}: z_{1}(\zeta(x))=\cdots=\right.$ $\left.z_{N-2}(\zeta(x))=0\right\}$ is everywhere 1-dimensional, it follows from theorem 4 that $\hat{A}-\zeta^{-1}\left(E_{2}\right)$ is Stein. Hence $A-E_{2}=\zeta\left(\hat{A}-\zeta^{-1}\left(E_{2}\right)\right)$ itself is Stein.

Since $p \in E_{2}$ is the unique singular point of $\mathrm{A}$, then $U \cap V \cap D$ is Stein being a domain of holomorphy in the Stein manifold $A-E_{2}$.

If $p \in \partial D-Y$, then there exists $\mathrm{j}$ such that $p \notin Z_{j}$. We can find a Stein open neighborhood $\mathrm{U}$ of $\mathrm{p}$ in $\mathrm{X}$ such that $U \cap Z_{j}=\emptyset$. Then $U \cap D=U \cap\left(D-Z_{j}\right)$ is Stein.

The main step in the proof of theorem 3 is to show, when $\mathrm{D}$ is, in addition, relatively compact in $\mathrm{X}$, that for all $p \in \partial D$, there exist an open neighborhood $\mathrm{U}$ of $\mathrm{p}$ in $\mathrm{X}$ and an exhaustion function $\mathrm{f}$ on $D \cap U$ such that for each open $V \subset \subset U$ there is a continuous function $g$ on 
$\mathrm{V}$ which is locally the maximum of a finite number of stricly plurisubharmonic functions with $|f-g|<1$. Which implies that $D \cap U$ is 1-complete with corners.

(A result due to Peternell [12]).

This result will be applied in connection with

the Diederich-Fornaess theorem [6] which asserts that an irreducible $\mathrm{n}$-dimensional complex space $\mathrm{X}$ is Stein if $\mathrm{X}$ is 1-complete with corners.

The proof is also based on the following result of M.Peternell [12]

Lemma 1. -Let $\mathrm{X}$ be a complex space of pure dimension $\mathrm{n}$, $W \subset X \times X$ be an open set and $f \in F_{n}\left(W-\Delta_{X}\right)$ where $\Delta_{X}=\{(x, x): x \in X\}$, and let $S \subset \subset S^{\prime} \subset \subset X$ be open subsets of $\mathrm{X}$ such that $S \times S^{\prime} \subset \subset W$. Define $s(x)=\operatorname{Sup}\left\{f(x, y): y \in \overline{S^{\prime}}-S\right\}$ for $x \in S$ and assume that $s(x)>f(x, y)$ if $y \in \partial S^{\prime}$.

If $\mathrm{S}$ is Stein, then for each $D \subset \subset S$ and each $\varepsilon>0$, there is a $g \in F_{1}(D)$ such that $|g-s|<\varepsilon$ on D.

Here $F_{n}(D)$ and $F_{1}(D)$ denote respectively the sets of continuous functions on $\mathrm{D}$ which are locally the maximum of a finite number of strongly n-convex (resp. stricly psh) functions.

Proof of theorem 3 Clearly we may suppose that D is relatively compact in X.

Since the Stein property is invariant under normalization [11], we may assume that $\mathrm{X}$ is normal and connected.

For $n=2$, theorem 3 follows as an immediate consequence of theorem 2. Then we may also assume that $n \geq 3$.

Let $p \in \partial D \cap Y$, and choose a Stein open neighborhood $\mathrm{U}$ of $\mathrm{p}$ in $\mathrm{X}$ that can be realized as a closed complex subspace of a domain $M$ in $\mathbb{C}^{N}$.

Let $\mathrm{E}$ be a complex affine subspace of $\mathbb{C}^{N}$ of maximal dimension such that $\mathrm{p}$ is an isolated point of $E \cap U$, and let $\mathrm{E}$ ' be any complementary complex affine subspace to $\mathrm{E}$ in $\mathbb{C}^{N}$ through p.

We may choose the coordinates $z_{1}, \cdots, z_{N}$ and the space $E^{\prime}$ such that $z_{i}(p)=0$ for all $i \in\{1, \cdots, N\}$ and $\operatorname{dim}\left(E^{\prime} \cap U\right) \geq 1$. Since $T=E^{\prime} \cap U$ is a closed analytic set in $\mathrm{U}$, and $h(z, w)=|z|^{2}+|w|^{2}-\log \left(|z-w|^{2}\right)$ a strongly n-convex $C^{\infty}$ function on $E^{\prime} \times E^{\prime}-\Delta_{E^{\prime}}$, then there exists a strongly n-convex $C^{\infty}$ function $\psi$ on a neighborhood $\mathrm{W}$ of $T^{\prime}=$ 
$T \times T-\Delta_{T}$ with $W \subset U \times U-\Delta_{U}$ such that $h \leq \psi / T^{\prime} \leq h+1$. (See Demailly [5]).

Let $W^{\prime}$ be an open set in $U \times U$ such that $W=W^{\prime}-\Delta_{U}$. We may choose W'such that there exist a neighborhood $\mathrm{N}$ of $\mathrm{p}$ in $\mathrm{X}$ and a Stein open neighborhood $U_{1}$ of $\mathrm{p}$ with $U_{1} \subset \subset N$ and such that $U_{1} \times\left(N-U_{1}\right) \subset \subset W^{\prime}$.

We now construct an exhaustion function $f_{1}$ on $U_{1} \cap D$ such that for each open $Z \subset \subset U_{1} \cap D$ there is a $g \in F_{1}(Z)$ with $\left|g-f_{1}\right|<1$.

Let $f_{1}(z)=\operatorname{Sup}\left\{\psi(z, w), w \in \bar{N}-U_{1} \cap D\right\}, z \in U_{1} \cap D$

Obviously $f_{1}$ is an exhaustion function on $U_{1} \cap D$. There exists $m \geq 1$ such that $Z \subset \subset U_{1} \cap D_{m}$.

We now define

$$
g_{j}(z)=\operatorname{Sup}\left\{\psi(z, w): w \in \bar{N}-U_{1} \cap D_{j}\right\}, \text { for } z \in U_{1} \cap D_{j}, j \geq m
$$

Since $U_{1} \cap D_{j}$ is Stein, $\psi(z, w)$ is n-convex on $\mathrm{W}$, and $g_{j}(z)>\psi(z, w)$ for every $(z, w) \in\left(U_{1} \cap D_{j}\right) \times \partial N$, then there is a $h_{j} \in F_{1}(Z)$ such that $\left|g_{j}-h_{j}\right|<\frac{1}{2}$. Since, obviously, $\left(g_{j}\right)_{j \geq 1}$ converges uniformally on compact sets to $f_{1}$, then there is a $j \geq m$ such that $\left|g_{j}-f_{1}\right|<\frac{1}{2}$ on Z. Hence $\left|f_{1}-h_{j}\right|<1$. Now the theorem follows from the lemma and the theorem of Diederich-Fornaess [6]

\section{References}

[1] . A. Andreotti and R. Narasimhan. Oka's Heftungslemma and the Levi problem for complex spaces. Trans. AMS III, pp. 345366, (1964).

[2] . Behnke.H,Stein,K.: Konvergente Folgen Von Regularitatsbereichen and die Meromorphiekonvexitat, Math. Ann. 166, pp. 204-216, (1938)

[3] . M. Coltoiu,Remarques sur les réunions croissantes d'ouverts de Stein C. R. Acad. Sci. Paris. t. 307, Série I, pp. 91-94, (1988).

[4] . M. Coltoiu, Open problems concerning Stein spaces. Revue Roumaine de Mathématiques Pures et Appliquées. 
[5] . Demailly, J. P.: Cohomology of q-convex spaces in top degrees. Math. Z 204, pp. 283-295, (1990).

[6] . Diederich, H., Fornaess, J. E.: Smoothing q-convex functions in the singular case. Math. Ann. 273, pp. 665-671, (1986).

[7] . Fornaess, J. E.: An increasing sequence of Stein manifolds whose limit is not Stein, Math. Ann.223, pp. 275-277, (1976).

[8] . Fornaess J. E., Narasimhan. R.: The levi problem on complex spaces with singularities. Math. Ann. 248, pp. 47-72, (1980).

[9] . Grauert, H., Remmert, R.: Singularitaten Komplexer Manngifaltigkeiten und Riemannsche Gebiete. Math. Z. 67, pp. 103-128, (1957).

[10] . Markoe, A.: Runge Families and Inductive limits of Stein spaces.Ann. Inst. Fourier 27, Fax. 3 (1977).

[11] . Narasimhan, R.: A note on Stein spaces and their normalizations. Ann Scuela Norm. Sup. Pisa 16, pp. 327-333, (1962).

[12] . Peternell, M.: Continuous q-convex exhaustion functions. Invent. Math. 85, pp. 246-263, (1986).

[13] . Stein,K.: Uberlagerungen holomorph-vollstandiger Komplexer Raume.Arch.Math. 7, pp. 354-361, (1956).

[14 . Skoda,H.:Application de techniques $L^{2}$ la théorie des idéaux d'une algébre de fonctions holomorphes avec poids. Ann.Sci.Ecole Norm.Sup. Paris 5, pp. 545-579, (1972).

[15 . Simha,R.: On the complement of a curve on a Stein space. Math. Z. 82, pp. 63-66, (1963).

Received : March 2000. 


\author{
Alaoui Youssef \\ Institut Agronomique et Vétérinaire Hassan II \\ B. P. 6202, Instituts \\ Rabat 10101 \\ Maroc \\ e-mail : y.alaoui@iav.ac.ma
}

